\section{Comportamento relativo à exposição e proteção solar na população de 15 anos ou mais de 15 capitais brasileiras e Distrito Federal, 2002-2003}

\author{
Behaviors related to sunlight exposure versus \\ protection in a random population sample from \\ 15 Brazilian State capitals and the Federal District, \\ 2002-2003
}

\section{Abstract}

This article examines region-specific relations between prevalence of protection against sunlight and socio-demographic and behavioral variables in Brazil. Data were derived from a cross-sectional population-based random sample. Information on sunlight exposure was available for a total of 16,999 individuals 15 years and older. Comparing the North and South of Brazil, crude differences between women and men in the use of "sunscreen" and "protective headwear" were $+10.9 \%$ (95\%CI: 7.1; 14.6) and $-11.6 \%$ (95\% CI: -17.0; -6.3) in the North and $+21.3 \%$ (95\% CI: 17.7; 24.9) and -16.0\% (95\%CI: $-20.2 ;-12.5)$ in the South. Adjusted differences by selected variables confirmed that women use more sunscreen protection and less headwear protection as compared to men in both the North and South, but the difference was not homogeneous by region (interaction term $p$ value $<0.05)$.

Sunlight; Sunscreening Agents; Gender

\author{
André Salem Szklo ${ }^{1}$ \\ Liz Maria de Almeida ${ }^{1}$ \\ Valeska Figueiredo 1 \\ José de Azevedo Lozana 1 \\ Gulnar Azevedo e Silva Mendonça 1 \\ Lenildo de Moura 2 \\ Moysés Szklo 3
}

\section{Introdução}

Vários estudos relacionam a exposição à radiação solar como uma das principais causas de câncer de pele tipo melanoma e não melanoma 1. Particularmente no Brasil, dentre todas as localizações, o câncer de pele não melanoma é o mais freqüente, apresentando taxas brutas de incidência estimadas, para o ano de 2006, de 68,1 e 68,9 por 100 mil entre homens e mulheres, respectivamente ${ }^{2}$. A percepção dos possíveis efeitos nocivos da exposição solar e da sua participação na cadeia causal de alguns processos degenerativos e/ou mutagênicos 3 levou à realização de numerosas investigações, cujos resultados sugeriram que a exposição solar é também fator de risco para outras neoplasias e doenças, como, por exemplo, câncer de lábio ${ }^{1}$ e catarata senil 1 .

As medidas de exposição à radiação ultravioleta estão relacionadas tanto com características individuais quanto com fatores ambientais. Assim sendo, o tipo de câncer a ser desenvolvido parece ser função não somente do tipo de pele e fenótipo 4,5 e da história familiar 6 , mas também do nível de exposição cumulativa, sendo esse último intimamente relacionado com a latitude na qual se encontra o indivíduo 7 .

Hábitos de vida e proteção ao Sol podem mudar pelas variáveis citadas no parágrafo anterior, contribuindo, assim, de forma diferenciada para os níveis cumulativos de exposição ao Sol e sua relação sucessiva com determinados tipos de 
câncer ${ }^{8,9}$. Associadas a esses hábitos de vida e proteção, distinguem-se três situações de exposição solar: no lazer, no trabalho e na realização de atividades domésticas 10,11.

A utilização de bloqueadores solares como uma forma efetiva de proteção na redução da formação de lesões pré-cancerígenas e cancerígenas tem sido amplamente discutida na literatura. O efeito aparentemente protetor na redução de ceratoses não encontra consenso quando se estuda o seu efeito no câncer de pele melanoma. Apesar dessa controvérsia, protetores solares de amplo espectro, tais como os inorgânicos, são recomendados na prevenção de todas as neoplasias da pele, incluindo o melanoma 12,13. Outras formas de proteção recomendadas e que aparecem nos estudos sobre o tema são o uso de chapéus e o hábito de buscar a sombra quando no deslocamento ou permanência em áreas ensolaradas. No entanto, o efeito positivo oriundo da utilização de chapéus, por exemplo, ainda é contraditório na literatura 14,15,16.

Nos anos de 2002 e 2003, foi realizado, no Brasil, um inquérito domiciliar de base populacional sobre comportamentos de risco que, entre outros objetivos, avaliou as formas de proteção à radiação solar mais utilizadas nas cidades que participaram do estudo. Esse inquérito representou, assim, a primeira tentativa de obter-se, em nível nacional, informação com relação aos hábitos de proteção à exposição solar, contribuindo, portanto, para melhorar o conhecimento do nível de exposição solar cumulativa no Brasil e fornecer subsídios para programas de prevenção em nível nacional.

Neste artigo, procuramos caracterizar as diferenças encontradas no uso de formas de proteção à exposição solar mais utilizadas, a partir de dados do inquérito domiciliar realizado em capitais das cinco regiões brasileiras, segundo variáveis sócio-demográficas, particularmente gênero, e comportamentais.

\section{Metodologia}

\section{Desenho, procedimento e população de estudo}

O inquérito domiciliar sobre comportamentos de risco para doenças e agravos não transmissíveis foi um estudo transversal de base populacional realizado em 2002/2003, cuja população alvo foi composta por indivíduos com idade igual ou superior a 15 anos, residentes no Distrito Federal e em 15 capitais brasileiras. O modelo de amostragem adotado para a pesquisa foi o de uma amostra autoponderada com dois estágios de seleção. As unidades primárias de amostragem foram os setores censitários; e as unidades secundárias, os domicílios. Maiores detalhes sobre este estudo podem ser encontrados em recente publicação 17 .

O número de indivíduos que responderam, de forma completa, às perguntas sobre exposição e proteção à radiação solar foi de 16.999, sendo a sua distribuição por região mostrada na Tabela 1. O percentual médio de dados ausentes sobre os indivíduos selecionados para responderem sobre exposição à radiação solar e proteção à radiação solar foi de 10,3\%, variando de 5,3\% em Fortaleza a $16,3 \%$ no Rio de Janeiro.

\section{Coleta de dados}

A coleta dos dados ocorreu em dois períodos diferentes. Em 2002, foram visitadas Manaus, Belém, Fortaleza, João Pessoa, Belo Horizonte, Rio de Janeiro, Curitiba, Porto Alegre e o Distrito Federal. As coletas de dados em Natal, Recife, Aracaju, Vitória, São Paulo, Florianópolis e Campo Grande foram realizadas em 2003. Essa coleta não ocorreu de forma simultânea em todas as cidades, variando com relação ao período do ano e sua duração.

\section{Variáveis e instrumentos de aferição}

De interesse central para o presente artigo, o módulo cobrindo a exposição solar foi elaborado após a revisão do WHO Standard Risk Factor Questionnaire e Behavioral Risk Factor Surveillance System (BRFSS/Centers for Disease Control and Prevention). Esse módulo apresenta questões envolvendo exposição à radiação solar por, pelo menos, trinta minutos ("O Sr. fica em ambientes ensolarados por qualquer motivo, por exemplo, lazer/educação física, trabalho, atividades do lar ou quando está andando de um lugar para o outro por, pelo menos, 30 minutos seguidos, mesmo que de vez em quando?") e proteção entre indivíduos expostos a ambientes ensolarados por, pelo menos, trinta minutos ("Quando o $\mathrm{Sr}$. está em um ambiente ensolarado por mais de 30 minutos, com que freqüencia o Sr. usa protetor ou filtro solar?"; "Quando o Sr. está em um ambiente ensolarado por mais de 30 minutos, com que freqüência o Sr. fica na sombra?”; “Quando o Sr. está em um ambiente ensolarado por mais de 30 minutos, com que freqüência o Sr. usa chapéu com abas ou qualquer outro tipo de proteção para o rosto?"). As variáveis dicotômicas que definiram os diferentes tipos de proteção solar foram geradas a partir das perguntas citadas acima; para essas variáveis dicotômicas, a alternativa de resposta sempre foi comparada com todas as outras 
Proporções de exposição à radiação solar por, pelo menos, trinta minutos seguidos segundo variáveis selecionadas, estratificadas por região *

\begin{tabular}{|c|c|c|c|c|c|}
\hline \multirow[t]{2}{*}{ Variáveis selecionadas } & \multicolumn{5}{|c|}{ Proporção de indivíduos expostos à radiação solar (\%) } \\
\hline & Norte & Nordeste & Centro-Oeste & Sudeste & Sul \\
\hline \multicolumn{6}{|l|}{ Sexo ** } \\
\hline Homens & 73,5 & 75,8 & 81,2 & 81,5 & 82,7 \\
\hline Mulheres & $62,6 * \star \star$ & $62,9 * \star \star$ & $68,9 * \star \star$ & $69,6 * \star \star$ & $72,9 * \star *$ \\
\hline \multicolumn{6}{|l|}{ Idade (anos) \# } \\
\hline $15-24$ & 75,6 & 75,8 & 78,7 & 79,1 & 82,8 \\
\hline $25+$ & $63,5 * \star \star$ & $65,7 * \star \star$ & 72,4 *** & $73,3 * \star \star$ & $75,6 * \star \star$ \\
\hline \multicolumn{6}{|l|}{ Escolaridade (anos) \#\# } \\
\hline$<8$ & 66,4 & 67,5 & 79,0 & 71,4 & 73,0 \\
\hline$\geq 8$ & 68,4 & 69,8 & 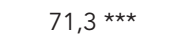 & 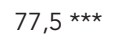 & $79,5 * \star \star$ \\
\hline \multicolumn{6}{|l|}{ Atividade física \#\#\# } \\
\hline Inativo & 55,5 & 57,7 & 61,3 & 65,4 & 66,8 \\
\hline Ativo & $75,5 * \star \star$ & 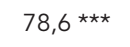 & $81,6 * \star \star$ & 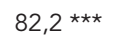 & $85,5^{\star \star \star \star}$ \\
\hline \multicolumn{6}{|l|}{$\mathrm{IMC}\left(\mathrm{kg} / \mathrm{m}^{2}\right) \S$} \\
\hline$<25$ & 69,5 & 70,5 & 73,9 & 76,1 & 79,0 \\
\hline$\geq 25$ & 65,0 & 69,6 & 76,8 & 74,2 & 74,1 \\
\hline
\end{tabular}

* O cálculo das proporções leva em consideração o setor censitário como Unidade Primária de Amostragem e o fator de expansão;

** O número de homens e mulheres amostrados e expandidos para as regiões Norte, Nordeste, Centro-Oeste, Sudeste e Sul é, respectivamente: 880 (Nexp $=649.020)$ e $1.144(\mathrm{Nexp}=852.498) ; 2.114(\mathrm{~N} \exp =1.503 .320)$ e $2.768(\mathrm{~N} \exp =1.970 .699), 868(\mathrm{~N} \exp =746.956)$ e $1.125(\mathrm{Nexp}=958.168), 2.174(\mathrm{Nexp}=$ $5.219 .923)$ e $2.824(\mathrm{Nexp}=6.953 .044), 1.400(\mathrm{Nexp}=857.480)$ e $1.702(\mathrm{Nexp}=1.056 .331)$;

*** Diferenças avaliadas segundo Pearson $\chi^{2}$, corrigido para efeito de desenho, e consideradas significativas com erro tipo I no nível de $5 \%$;

\# O número de indivíduos com idade inferior e superior a 25 anos amostrados e expandidos para as regiões Norte, Nordeste, Centro-Oeste, Sudeste e Sul é, respectivamente: $967(\mathrm{Nexp}=471.266)$ e $1.057(\mathrm{~N} \exp =1.030 .252) ; 1.703(\mathrm{~N} \exp =956.559)$ e $3.179(\mathrm{Nexp}=2.517 .460), 804(\mathrm{Nexp}=509.536)$ e 1.189 $(\mathrm{Nexp}=1.195 .588), 1.625(\mathrm{~N} \exp =3.014 .591)$ e $3.373(\mathrm{Nexp}=9.158 .376), 1.075(\mathrm{Nexp}=462.162)$ e $2.027(\mathrm{Nexp}=1.451 .649)$;

\#\# O número de indivíduos com baixo e alto grau de escolaridade (anos de estudos completos) amostrados e expandidos para as regiões Norte, Nordeste, Centro-Oeste, Sudeste e Sul é, respectivamente: $827(\mathrm{Nexp}=631.030)$ e $1.178(\mathrm{Nexp}=853.474) ; 2.117(\mathrm{Nexp}=1.515 .796)$ e $2.628(\mathrm{Nexp}=1.849 .459), 702$ $(\mathrm{N} \exp =611.864)$ e $1.235(\mathrm{~N} \exp =1.047 .648), 1.632(\mathrm{Nexp}=4.312 .667)$ e $3.216(\mathrm{Nexp}=7.453 .261), 903(\mathrm{Nexp}=569.315)$ e $2.084(\mathrm{Nexp}=1.269 .980)$; \#\# O número de indivíduos inativos e ativos amostrados e expandidos para as regiões Norte, Nordeste, Centro-Oeste, Sudeste e Sul é, respectivamente: $654(\mathrm{Nexp}=480.464)$ e $1.295(\mathrm{~N} \exp =953.072) ; 1.864(\mathrm{~N} \exp =1.344 .386)$ e $2.730(\mathrm{~N} \exp =1.904 .773), 669(\mathrm{Nexp}=4.392 .942)$ e $1.250(\mathrm{Nexp}=7.033 .934)$, $1.816(\mathrm{Nexp}=674.031)$ e $2.882(\mathrm{Nexp}=1.108 .123), 1.128(\mathrm{~N} \exp =682.499)$ e $1.789(\mathrm{Nexp}=1.108 .852)$;

$\S$ O número de indivíduos classificados com o Índice de Massa Corporal (IMC = peso(kg)/altura2 (m)) $<25$ ou $25+$ amostrados e expandidos para as regiões Norte, Nordeste, Centro-Oeste, Sudeste e Sul é, respectivamente: $1.100(\mathrm{Nexp}=780.686)$ e $366(\mathrm{Nexp}=316.962) ; 2.844(\mathrm{Nexp}=1.976 .238)$ e $968(\mathrm{Nexp}$ $=733.006), 1.343(\mathrm{~N} \exp =1.115 .564)$ e $416(\mathrm{Nexp}=389.580), 3.071(\mathrm{Nexp}=7.418 .284)$ e $1.256(\mathrm{Nexp}=3276.608), 2.124(\mathrm{Nexp}=1.277 .426)$ e $778(\mathrm{Nexp}$ $=535.592)$.

respostas possíveis (quase sempre, algumas vezes, raramente, nunca). Já para a variável dicotômica que definiu a exposição à radiação solar por, pelo menos, trinta minutos seguidos, as respostas possíveis eram do tipo sim ou não.

Para explorar a associação das duas variáveisdesfecho acima citadas com supostas variáveis prognósticas demográficas, sócio-econômicas e relacionadas com o comportamento, considerou-se, para cada desfecho, um modelo logístico no qual todas as variáveis foram incluídas simultaneamente no mesmo. Assim sendo, as variáveis que não apresentaram um efeito independente estatisticamente significativo nos dois modelos, considerando um erro tipo I de 5\%, foram excluídas do nosso estudo. As variáveis incluídas neste estudo foram, portanto: sexo, idade, escolaridade, atividade física, sobrepeso e região.

Atividade física foi definida como uma variável dicotômica em que indivíduos inativos (sedentários e insuficientemente ativos) foram comparados com os minimamente ativos e muito ativos, tal como especificado pelo International Physical Activity Questionnaire Scoring Protocol 18. Sobrepeso foi definido por uma variável dicotômica na qual indivíduos com índice de massa corporal (IMC) $\geq 25 \mathrm{~kg} / \mathrm{m}^{2}$ foram comparados com os de IMC $<25 \mathrm{~kg} / \mathrm{m}^{2}$. Os 
dados foram agrupados segundo as cinco regiões do país: Norte (Manaus e Belém), Nordeste (Fortaleza, Natal, Recife, João Pessoa e Aracaju); Sudeste (Vitória, Belo Horizonte, Rio de Janeiro e São Paulo); Centro-Oeste (Distrito Federal e Campo Grande); e Sul (Curitiba, Florianópolis e Porto Alegre), por meio do critério de homogeneidade com relação à proporção de utilização de protetor solar e chapéu apresentado na Figura 1. A variável escolaridade foi criada por uma combinação entre as respostas envolvendo última "série" e "grau" cursados completamente; o ponto de corte foi estabelecido entre ensino fundamental incompleto (menos de 8 anos de estudo) e ensino fundamental completo (8 anos ou mais de estudo).

\section{Análise de dados}

Foram estimadas proporções simples de exposição à radiação solar para sexo, idade, escolaridade, atividade física, estado nutricional e região, e, nos indivíduos expostos à radiação solar, proporções simples de proteção para os três tipos de proteção solar acima descritos para essas mesmas variáveis. Para avaliar as diferenças encontradas nas proporções entre as categorias das variáveis acima citadas, estratificadas por região, foi realizado um teste de Pearson $\chi^{2}$ corrigido para efeito de desenho ${ }^{19}$. Finalmente, foi realizada uma análise de regressão linear multivariada, estratificada por duas regiões que apresentam enormes diferenças com relação à latitude, tipo de pele dos indivíduos, e contextos econômicos e sócio-culturais (Norte e Sul), tendo como variável dependente a proteção solar e como variável independente o gênero, com controle por todas as co-variáveis mencionadas. Anteriormente, para o cálculo dos intervalos de confiança das diferenças ajustadas na proporção de uso de proteção solar por gênero, obtidas pelo modelo de regressão linear multivariado, considerou-se um erro tipo I de 5\%. A interação aditiva entre a região e o gênero foi também avaliada por meio de um modelo de regressão linear multivariado contendo o termo de interação. O uso do modelo aditivo para avaliação de interação deveu-se à importância dos resultados do ponto de vista de prevenção ${ }^{20}$. O processamento de variáveis e a análise de dados foram realizados utilizando-se o programa Stata 8.2 (Stata Corporation, College Station, Estados Unidos). Na análise, utilizou-se o comando svy do aplicativo para lidar adequadamente com a estrutura amostral de conglomeração e permitir a incorporação das frações de expansão nas análises 21 .

Figura 1

Proporçães de proteção à radiação solar (\%), estratificadas por região e por tipos de proteção solar.

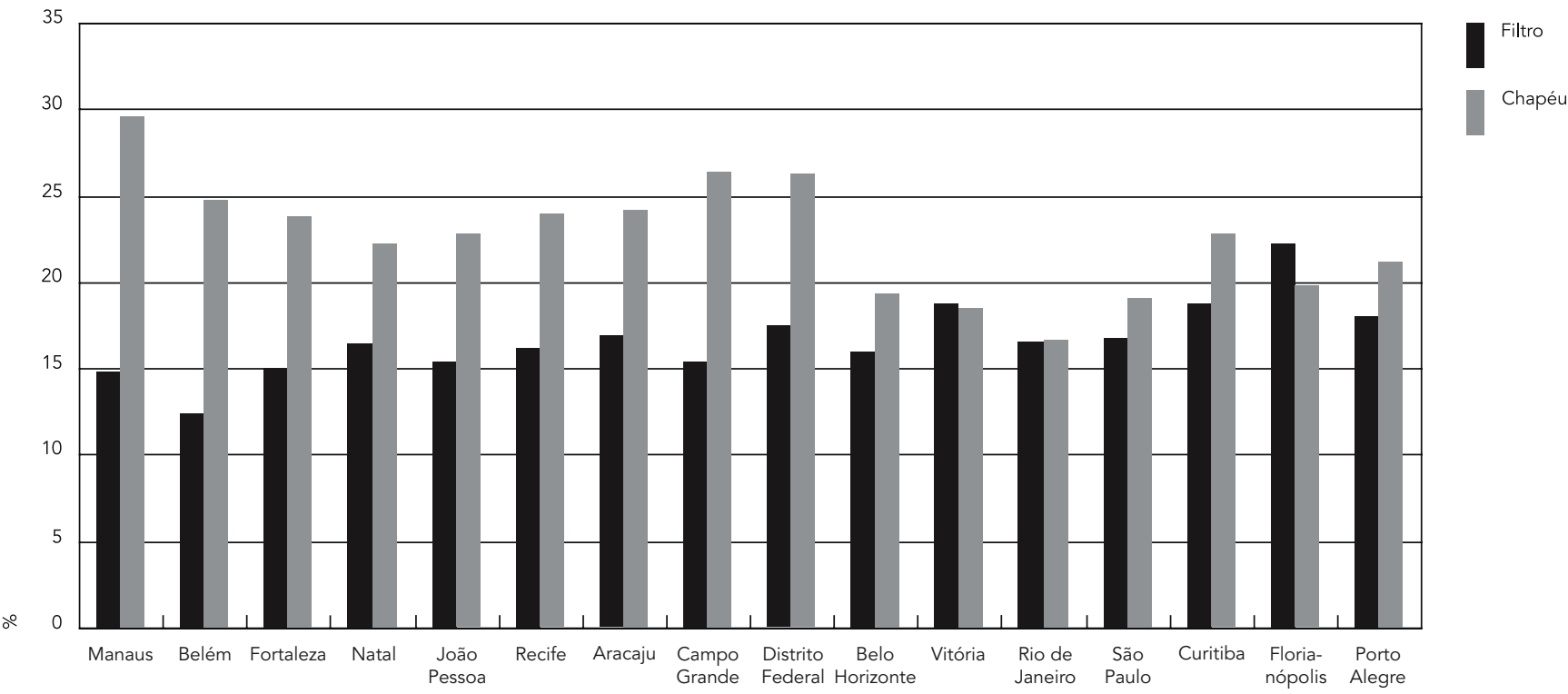




\section{Resultados}

A Figura 1 apresenta as proporções de utilização do protetor solar e chapéu estratificadas por todas as capitais pertencentes ao estudo. Podemos observar uma certa homogeneidade no interior das macrorregiões brasileiras. As maiores proporções de proteção com relação à utilização do filtro solar e do chapéu foram encontradas nos indivíduos pertencentes, respectivamente, a Florianópolis e a Manaus; já as menores proporções correspondem, respectivamente, às cidades de Belém e Rio de Janeiro.

Para todas as regiões representadas, uma maior proporção de exposição à radiação solar por, pelo menos, trinta minutos seguidos foi observada entre os homens e os indivíduos jovens quando comparados com as mulheres e com os indivíduos de 25 anos ou mais de idade, respectivamente $(p<0,05)$. Nas regiões Sul e Sudeste, os indivíduos com maior grau de escolaridade foram aqueles com maior proporção de exposição à radiação solar por, pelo menos, trinta minutos seguidos. Nas outras regiões, ou não foi observada nenhuma diferença ou, no caso da Região Centro-Oeste, foi observada uma situação inversa, ou seja, os indivíduos com menor escolaridade foram aqueles que apresentaram maior proporção de exposição à radiação solar por, pelo menos, trinta minutos seguidos. Nenhuma diferença significativa na exposição à radiação solar foi observada entre os indivíduos com sobrepeso (incluindo os obesos) e os com peso normal (incluindo os de baixo peso), porém, os dados sugerem que indivíduos fisicamente ativos estariam com uma maior proporção de exposição à radiação solar por, pelo menos, trinta minutos seguidos.

Os menores percentuais totais de exposição à radiação solar por, pelo menos, trinta minutos seguidos foram encontrados entre os indivíduos pertencentes às regiões Norte e Nordeste (Tabela 1).

Na Tabela 2, são apresentadas as freqüências relativas às três formas de proteção solar, por região, estratificadas pelas variáveis selecionadas. Em todas as regiões estudadas, observaram-se proporções mais elevadas de proteção com o filtro solar e procura pela sombra nas mulheres do que nos homens, enquanto o contrário foi observado com relação à proteção com o chapéu. Indivíduos de 25 anos ou mais de idade apresentaram percentuais de proteção maiores do que os de menos de 25 anos, independentemente da região e do tipo de proteção. Em todas as regiões, observou-se uma maior proporção de proteção com filtro solar entre indivíduos de maior escolaridade quando comparados com os de menor grau de escolaridade, enquanto que a proteção com o chapéu apresentou uma proporção maior entre os indivíduos de menor escolaridade. No que diz respeito à procura pela sombra, foi encontrada uma maior proporção de procura pela sombra entre os indivíduos de menor grau de escolaridade (quando comparados com os de maior grau de escolaridade), principalmente para as regiões Sul e Sudeste. As proporções de uso do filtro solar e da procura pela sombra foram maiores em indivíduos fisicamente inativos do que nos fisicamente ativos, ao passo que o oposto foi observado com relação ao uso do chapéu. Entre os indivíduos com índice de massa corpórea de $\geq 25 \mathrm{~kg} / \mathrm{m}^{2}$, foi observada uma menor proporção de uso do filtro solar quando comparados aos indivíduos de IMC $<25 \mathrm{~kg} / \mathrm{m}^{2}$, porém maior proporção de procura pela sombra e utilização de chapéu como forma de proteção solar.

A Tabela 3 apresenta os dados brutos e ajustados da relação entre gênero e os três tipos de proteção solar para as regiões Norte e Sul. As mulheres apareceram com uma maior proporção de utilização do filtro solar como proteção do que os homens, e essa relação parece ter sido modificada por região: no Sul do país, a diferença por gênero na proteção pelo uso do filtro solar foi maior do que a verificada na região Norte (termo de interação aditiva: $p=0,003$ ). Por outro lado, as maiores proporções de uso de chapéu foram referidas pelos homens quando comparados às mulheres, e essa relação também foi modificada por região: no Sul do país, a diferença por gênero na proteção solar com o uso do chapéu foi maior do que a verificada na Região Norte (termo de interação aditiva: $p=0,050$ ). Com relação à proteção pela procura de sombra, as mulheres apresentaram uma maior proporção dessa proteção do que os homens, e a associação entre gênero e a procura pela sombra não foi modificada por região.

\section{Discussão}

Os homens e os jovens foram os que apresentaram as maiores prevalências de exposição à radiação solar por, pelo menos, trinta minutos, em todas as regiões brasileiras. Esses dados estão em conformidade com a literatura que aponta os homens como tendo menor preocupação e conhecimento sobre os efeitos nocivos relacionados à radiação ultravioleta, assim como aqueles mais freqüentemente empregados em atividades ocupacionais relacionadas à exposição a esse tipo de radiação 10,22,23,24,25. Os jovens também constituem um grupo vulnerável aos efeitos nocivos do Sol, tanto pelo aspecto estético do 
Proporções de proteção à radiação solar (\%) segundo variáveis selecionadas, estratificadas por região e por tipos de proteção solar *.

\begin{tabular}{|c|c|c|c|c|c|}
\hline Variáveis selecionadas & Norte & Nordeste & Centro-Oeste & Sudeste & Sul \\
\hline \multicolumn{6}{|l|}{ Filtro } \\
\hline \multicolumn{6}{|l|}{ Sexo } \\
\hline Homens & 7,9 & 8,0 & 8,2 & 8,0 & 7,5 \\
\hline Mulheres & 18,8 ** & 23,0 ** & 22,7 ** & 24,3 ** & 28,8 ** \\
\hline \multicolumn{6}{|l|}{ Idade (anos) } \\
\hline $15-24$ & 10,6 & 11,9 & 8,3 & 12,0 & 11,9 \\
\hline $25+$ & $15,3 \star \star$ & $17,5 * \star$ & 19,3 ** & 18,3 ** & 20,8 ** \\
\hline \multicolumn{6}{|l|}{ Escolaridade (anos) } \\
\hline$<8$ & 8,6 & 7,3 & 8,5 & 8,4 & 10,9 \\
\hline$\geq 8$ & 16,9 ** & 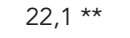 & 20,6 ** & 20,8 ** & 21,6 ** \\
\hline \multicolumn{6}{|l|}{ Atividade física } \\
\hline Inativo & 15,7 & 18,4 & 17,1 & 16,4 & 20,7 \\
\hline Ativo & 13,2 & $14,7 \star \star$ & 15,4 & 17,2 & 18,3 \\
\hline \multicolumn{6}{|l|}{ 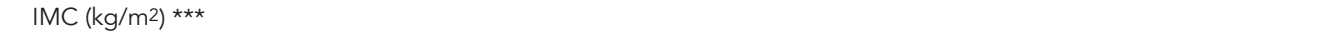 } \\
\hline$<25$ & 15,6 & 18,2 & 17,4 & 17,6 & 19,1 \\
\hline$\geq 25$ & 13,5 & 16,5 & 13,8 & 16,5 & 18,6 \\
\hline \multicolumn{6}{|l|}{ Chapéu } \\
\hline \multicolumn{6}{|l|}{ Sexo } \\
\hline Homens & 33,4 & 33,6 & 37,4 & 24,8 & 30,4 \\
\hline Mulheres & $21,8 * \star$ & $14,8 * \star$ & $16,0 * \star$ & $12,8 * \star$ & 14,0 ** \\
\hline \multicolumn{6}{|l|}{ Idade (anos) } \\
\hline $15-24$ & 22,1 & 13,8 & 19,8 & 14,0 & 15,8 \\
\hline $25+$ & 30,1 ** & 28,2 ** & 29,2 ** & 19,9 ** & 24,0 ** \\
\hline \multicolumn{6}{|l|}{ Escolaridade (anos) } \\
\hline$<8$ & 32,9 & 26,2 & 33,4 & 18,9 & 28,3 \\
\hline$\geq 8$ & 23,3 ** & 22,2 ** & 21,4 ** & 17,9 & 19,4 ** \\
\hline \multicolumn{6}{|l|}{ Atividade física } \\
\hline Inativo & 25,1 & 22,1 & 18,0 & 16,1 & 19,5 \\
\hline Ativo & 27,7 & 24,4 & 29,6 ** & 19,7 ** & 22,1 \\
\hline \multicolumn{6}{|l|}{$\operatorname{IMC}\left(\mathrm{kg} / \mathrm{m}^{2}\right)^{\star \star \star}$} \\
\hline$<25$ & 28,2 & 23,8 & 26,1 & 19,5 & 21,4 \\
\hline$\geq 25$ & 34,7 & 29,9 ** & 29,1 & 17,9 & 23,7 \\
\hline \multicolumn{6}{|l|}{ Sombra } \\
\hline \multicolumn{6}{|l|}{ Sexo } \\
\hline Homens & 35,4 & 35,8 & 32,4 & 28,9 & 20,7 \\
\hline Mulheres & 43,4 ** & 41,3 ** & $42,9 * \star$ & 32,9 ** & 27,8 ** \\
\hline \multicolumn{6}{|l|}{ Idade (anos) } \\
\hline $15-24$ & 34,9 & 33,2 & 32,9 & 25,7 & 18,2 \\
\hline $25+$ & 42,2 ** & 41,1 ** & 40,2 ** & 32,9 ** & $26,5^{\text {** }}$ \\
\hline \multicolumn{6}{|l|}{ Escolaridade (anos) } \\
\hline$<8$ & 41,3 & 39,3 & 40,7 & 35,7 & 28,6 \\
\hline$\geq 8$ & 38,2 & 38,2 & 36,3 & 28,3 ** & 22,6 ** \\
\hline \multicolumn{6}{|l|}{ Atividade física } \\
\hline Inativo & 42,2 & 40,9 & 44,0 & 30,7 & 28,5 \\
\hline Ativo & 38,6 & 36,4 ** & 34,7 ** & 30,5 & 22,1 ** \\
\hline \multicolumn{6}{|l|}{ 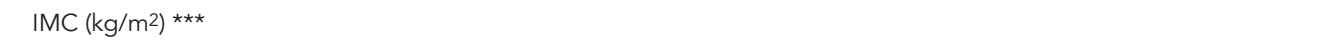 } \\
\hline$<25$ & 40,4 & 38,3 & 36,8 & 30,2 & 24,1 \\
\hline$\geq 25$ & 41,9 & 40,7 & 37,9 & 32,2 & 25,0 \\
\hline
\end{tabular}

* O cálculo das proporções leva em consideração o setor censitário como Unidade Primária de Amostragem e o fator de expansão; o número de indivíduos expandidos para as regiões Norte, Nordeste, Centro-Oeste, Sudeste e Sul para cada categoria das variáveis estudadas pode ser obtido pela Tabela 1;

** Diferenças avaliadas segundo Pearson $\chi^{2}$, corrigido para efeito de desenho, e consideradas significativas com erro tipo I no nível de $5 \%$;

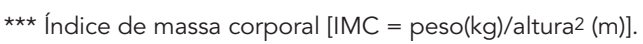


Diferenças brutas e ajustadas * nas proporções de proteção à radiação solar segundo sexo, estratificadas pela região e pelos tipos de proteção solar.

\begin{tabular}{|c|c|c|c|c|c|}
\hline \multirow[t]{2}{*}{$\begin{array}{l}\text { Variáveis } \\
\text { selecionadas }\end{array}$} & \multicolumn{2}{|c|}{$\begin{array}{l}\text { Diferenças brutas } \\
\text { \% (IC95\%) }\end{array}$} & \multicolumn{2}{|c|}{$\begin{array}{c}\text { Diferenças ajustadas } \\
\text { \% (IC95\%) }\end{array}$} & \multirow[t]{2}{*}{$\begin{array}{l}\text { Interação entre sexo } \\
\text { e região (valor de } p \text { ) }\end{array}$} \\
\hline & Norte & Sul & Norte & Sul & \\
\hline \multicolumn{6}{|l|}{ Filtro solar } \\
\hline Homens & Referência & Referência & Referência & Referência & Referência \\
\hline Mulheres & $10,9(7,1 ; 14,6)$ & $21,3(17,7 ; 24,9)$ & $12,9(8,3 ; 17,4)$ & $22,2(18,2 ; 26,3)$ & 0,003 \\
\hline \multicolumn{6}{|l|}{ Chapéu } \\
\hline Homens & Referência & Referência & Referência & Referência & Referência \\
\hline Mulheres & $-11,7(-17,0 ;-6,3)$ & $-16,3(-20,3 ;-12,4)$ & $-6,5(-10,1 ;-0,1)$ & $-14,2(-18,2 ;-10,2)$ & 0,050 \\
\hline \multicolumn{6}{|l|}{ Sombra } \\
\hline Homens & Referência & Referência & Referência & Referência & Referência \\
\hline Mulheres & $8,0(1,8 ; 14,2)$ & $7,1(3,3 ; 10,9)$ & $10,2(3,2 ; 17,1)$ & $6,6(2,6 ; 10,6)$ & 0,349 \\
\hline
\end{tabular}

* Análise de regressão linear multivariada, estratificada por duas regiões (Norte e Sul), tendo como variável dependente a proteção solar e como variável independente o sexo, controlada por escolaridade, idade, atividade física e sobrepeso, levando em consideração o setor censitário como Unidade Primária de Amostragem e o fator de expansão; o número de indivíduos expandidos para as regiões Norte e Sul para homens e mulheres pode ser obtido pela Tabela 1 ; para o cálculo dos intervalos de confiança das diferenças brutas e ajustadas na proporção de uso de proteção solar por gênero, considerou-se um erro tipo I de $5 \%$.

bronzeado $26,27,28$ quanto pela maior freqüência de atividade física, sobretudo relacionada à prática de esportes 29 .

O papel do nível sócio-econômico com relação à exposição à radiação ultravioleta é importante, já que essa variável pode determinar diferentes combinações de exposição e uso de medidas de proteção que variam por região 30,31 . Na Tabela 1, observou-se que, nas regiões mais desenvolvidas como Sul e Sudeste, os indivíduos de maior grau de escolaridade foram aqueles mais freqüentemente expostos à radiação solar, enquanto que, na Região Centro-Oeste, os indivíduos de menor grau de escolaridade foram os mais expostos à radiação solar. No nosso estudo, existiam duas perguntas que procuravam saber se o indivíduo tinha se exposto à radiação solar por, pelo menos, trinta minutos em atividades ocupacionais ou de lazer. Para os indivíduos de maior grau de escolaridade, em todas as regiões, a contribuição relativa da exposição à radiação solar em atividades de lazer foi aproximadamente quatro vezes superior àquelas relacionadas ao trabalho. Já entre os indivíduos de menor grau de escolaridade, houve um certo equilíbrio entre essas duas categorias de exposição, com exceção do Centro-Oeste onde a exposição à radiação solar no trabalho foi duas vezes superior àquela verificada nas atividades de lazer (dado não mostrado). É possível, portanto, que, nas regiões Sul e Sudeste, a contribuição da exposição solar nesses indivíduos seja devida, de forma mais importante, a atividades de lazer, enquanto que, na Região Centro-Oeste, possa existir uma maior contribuição do tipo de ocupação. Essas considerações merecem um estudo mais detalhado a respeito.

A maior proporção encontrada de indivíduos fisicamente ativos expostos à radiação solar sugere que a atividade física ocorreu principalmente em áreas abertas para as várias situações de exposição (lazer, trabalho, atividade doméstica). Os dados de IMC, apesar de não serem estatisticamente significativos, possivelmente refletiram a relação inversa existente com a intensidade de atividade física, na qual, em média, quanto maior o grau de atividade física, menor o índice de massa corpórea (dado não mostrado: $\beta$ linear $=-0,61$; $\mathrm{p}<0,001)$.

Os dados de proteção solar, brutos e ajustados, segundo gênero, coincidem com os resultados encontrados em outros estudos internacionais realizados $8,10,32,33$, nos quais se verificou que o uso de filtro solar foi mais comum entre mulheres, enquanto o uso de chapéus predominou entre homens. A literatura é farta em trabalhos que revelam que as mulheres, de uma maneira geral, cuidam de sua saúde numa freqüência maior do que os homens. No caso dos cuidados com a pele, associam-se, provavelmente, questões de ordem estética 24,34. Esses fatores poderiam explicar uma maior freqüência de uso do filtro solar e uma maior procura pela sombra nesse grupo. Já o uso do chapéu, mais freqüente en- 
tre os homens, pode mostrar que esses também acabaram, de alguma maneira, protegendo-se da exposição solar com um método que, provavelmente, estaria mais ligado a questões culturais. Todas essas hipóteses merecem ser aprofundadas em estudos futuros.

No nosso estudo, observamos menor proteção nos indivíduos jovens e maior nível de exposição à radiação solar nessa faixa etária. Como o número de episódios de queimadura por exposição solar na infância parece ser um fator de risco tanto para o câncer de pele melanoma quanto para o não melanoma $4,5,35$, esse grupo etário deveria ser alvo de prevenção primária com relação a esses tipos de câncer. Diversos estudos já foram conduzidos nesse sentido $36,37,38$ e espera-se que os resultados, a médio e longo prazo, sejam relevantes. É muito importante, contudo, implementar essas estratégias de prevenção tendo em mente, ao mesmo tempo, que a exposição solar tem efeitos benéficos como, por exemplo, garantir o suprimento de vitamina D necessário 39, prover relaxamento e bem-estar 40 e aumentar a auto-estima 41 .

O papel da escolaridade como proxy do nível sócio-econômico faz com que esta variável possa discriminar diferentes combinações de medidas de proteção que variam por região. Enquanto o uso do filtro solar reflete, com mais intensidade, o custo envolvido com a aquisição do mesmo para todas as regiões, o uso do chapéu e a procura pela sombra levam, provavelmente, mais em consideração os aspectos sócio-culturais ${ }^{30}$.

A prática da atividade física, provavelmente mais voltada à ocupação e à prática de esportes em áreas abertas, pareceu estar mais relacionada ao uso do chapéu como forma de proteção do que ao uso do filtro solar ou à procura pela sombra. Vale a pena ressaltar que, apesar de não serem estatisticamente significativas, as diferenças na utilização das formas de proteção solar segundo o nível de atividade física refletiram a maior contribuição dos jovens para a parcela de indivíduos fisicamente ativos (dados não mostrados). Esse grupo, por sua vez, mostrou uma menor freqüência do uso de formas de proteção. Assim sendo, a prática de atividades esportivas em áreas abertas, por exemplo, representa um elemento importante a ser considerado no momento de se estabelecer uma estratégia de prevenção primária eficaz 42,43 .

O IMC aumenta com a idade, o que sugere que os achados das freqüências de utilização de formas de proteção segundo categorias de IMC acompanhariam os resultados encontrados para as categorias de idade. Entretanto, não foi essa a direção dos achados para o uso do filtro solar. Uma possível explicação seria uma combinação de fatores, tais como menor cuidado com a saúde e maior dificuldade de aplicação do filtro solar. Essa hipótese foi reforçada quando refizemos as análises apenas para os indivíduos obesos (IMC $\geq$ $30 \mathrm{~kg} / \mathrm{m}^{2}$ ), com idade mais avançada e/ou maior comportamento de risco. Os resultados fortaleceram a menor freqüência de utilização do filtro solar entre esses indivíduos, como apontam outros estudos 10,44.

Ao compararmos as proporções de utilização das proteções solares segundo gênero entre as regiões Norte e Sul do país, verificamos que, para os homens, não foram observadas diferenças regionais com relação à proteção pelo filtro solar e proteção pelo uso de chapéu; entre as mulheres, contudo, após ajuste pelas variáveis sócio-demográficas e de comportamento (dado não mostrado), as diferenças brutas regionais que existiam permaneceram tanto para proteção com o filtro solar quanto para aquela com o uso do chapéu. Particularmente com relação à procura pela sombra, percebe-se que tanto os dados brutos quanto os ajustados indicaram uma maior proporção de indivíduos (ambos os sexos) protegidos localizados na Região Norte. Esses resultados sugerem que as diferenças regionais observadas podem estar relacionadas com a latitude 45 e o tipo de pele dos indivíduos 46 e devem ser objeto de estudos futuros com avaliação de contextos econômicos e sócio-culturais.

No Brasil, os índices, tanto de radiação UVA quanto de radiação UVB, variam muito ao longo das regiões. Enquanto a radiação UVB decresce à medida que se distancia da Linha do Equador, as maiores concentrações de radiação UVA são encontradas entre os paralelos $20^{\circ}$ e $40^{\circ} 47$. Essa variação dos níveis de radiação solar ao longo das capitais pode ter a sua influência neste complexo mecanismo de percepção do risco que envolve provavelmente tanto questões de susceptibilidade individual quanto valores e crenças sócioculturais 41 . Essa maior ou menor percepção do risco, aliada a questões de ordem econômica, determinaria o comportamento com relação à proteção solar ${ }^{48}$. Assim sendo, poderíamos dizer que esses fatores independentes interagiriam entre si potencializando ou atenuando alguns efeitos da exposição solar com relação aos seus desfechos mórbidos 7 .

As capitais da Região Sul, quando comparadas com as do Norte, apresentam as maiores taxas brutas estimadas de incidência de câncer de pele melanoma $(8,7$ por 100 mil habitantes na Região Sul e 1,3 por 100 mil habitantes na Região Norte) e não melanoma (77,4 por 100 mil habitantes na Região Sul e 73,5 por 100 mil habitantes na Região Norte) ${ }^{3}$, supondo mínimo o efeito do sub-registro diferenciado por região. 
Essas maiores taxas de incidência, fruto de uma interação de diversos fatores individuais-ambientais já abordados ao longo deste texto, podem levar a uma maior percepção coletiva do risco e a uma procura maior pela utilização de filtros solares, tal como foi observado na Região Sul. É importante assinalar que não foi objetivo deste estudo obter uma história individual de exposição e proteção com relação à radiação solar ao longo da vida, o que permitiria comparar efeitos protetores a curto ou a longo prazo. Os nossos resultados, conseqüentemente, referem-se a medidas de proteção utilizadas, corretamente ou não (o efeito positivo do bloqueador solar pode ser reduzido ou abolido no caso de utilização inadequada do mesmo 49,50, por esses indivíduos na época em que as entrevistas foram realizadas.

No que diz respeito à distribuição fenotípica brasileira, segundo dados do Instituto Brasileiro de Geografia e Estatística (IBGE) 51, existe uma grande heterogeneidade ao longo das latitudes do território brasileiro, chegando a aproximadamente $89 \%$ de população de raça branca nas áreas urbanas dos Estados de Santa Catarina e Rio Grande do Sul, onde a imigração de descendentes de europeus é muito forte (alemães, poloneses, italianos) e apenas a $28 \%$ nas áreas urbanas dos Estados do Amazonas e Pará. Entretanto, devido à grande mistura racial da população brasileira, as informações referidas de cor da pele mereceriam ser aprofundadas 52 .

A maior parte das capitais brasileiras encontra-se perto do litoral, e as diversas situações de exposição (lazer, trabalho, atividade doméstica) podem ter influências importantes nos níveis de exposição solar cumulativos 11. Conseqüentemente, ao juntarmos diversas capitais por região, podemos estar juntando cidades com características diferentes de situação de exposição solar (por exemplo, capitais localizadas perto do litoral e no interior). No entanto, ao excluirmos a única cidade do Sul do país que se encontra no litoral (Florianópolis), os resultados das comparações entre as duas regiões permaneceram virtualmente idênticos.

A estação do ano em que a informação foi coletada pode ter influenciado os resultados apresentados, dado que a percepção do desconforto com relação à exposição solar pode ter sido mediada provavelmente pelas condições ambientais presentes na época da entrevista. Para verificarmos essa hipótese, estratificamos as capitais do Norte e do Sul entre aquelas que tiveram as entrevistas realizadas no período que incluiu o mês de maior radiação diária média (http://www.cresesb.cepel.br/abertura.htm, acessado em 10/Abr/2006) e aquelas que não envolveram esse período, criando uma variável dicotômica que foi incluída no modelo. Os resultados, entretanto, permaneceram praticamente inalterados.

As perdas de informação relacionadas aos dados ausentes dos indivíduos selecionados também podem ter influenciado os resultados apresentados. Ao compararmos o grupo de indivíduos que se recusou a responder e aquele que forneceu informações (dado não mostrado), observamos uma maior proporção de homens entre aqueles que recusaram para todas as capitais. Com relação à escolaridade, apenas Curitiba e Porto Alegre apresentaram uma maior proporção de indivíduos de maior escolaridade entre aqueles pertencentes ao grupo que se recusou. Finalmente, em relação à faixa etária, na maioria das capitais, não se observou diferença etária entre os grupos analisados, com exceção de Rio de Janeiro, Curitiba e Distrito Federal, onde a recusa foi maior entre os indivíduos com 25 anos ou mais anos de idade. Assim sendo, se assumirmos que os indivíduos que se perderam têm as mesmas características de comportamento daqueles que permaneceram, provavelmente, os resultados da nossa população devem estar subestimados com relação à proporção total de exposição à radiação solar e utilização do chapéu e superestimados para a proporção total de utilização de filtro solar e procura pela sombra (diferenças observadas por gênero). Além disso, podemos dizer que o percentual médio de perdas foi de $10 \%$, e que as capitais das regiões Sul e Norte não ficaram muito distanciadas desse percentual, fazendo com que os resultados das análises comparativas sejam, provavelmente, conservadores.

Os dados apresentados aqui para as regiões brasileiras devem ser generalizados com cautela, pois, nem todas as capitais foram pesquisadas e tampouco as cidades do interior das regiões. No caso da Região Norte, somente duas capitais fizeram parte do estudo - Manaus e Belém -, que são, por outro lado, as cidades mais populosas da região. Na Região Centro-Oeste, a cautela deve ser maior porque apenas as cidades de Campo Grande e o Distrito Federal foram pesquisadas.

Neste artigo, enfatizamos os tipos de proteção solar que refletiram percepção de risco e de desconforto com relação aos efeitos nocivos do Sol. Apesar de existirem estudos científicos apontando para outras formas de proteção baseadas em aspectos endógenos, ou seja, os aspectos protetores conferidos pelos hábitos alimentares, em que micronutrientes podem funcionar como absorvedores de exposição ou antioxidantes 53,54, a proteção pelo uso de roupas adequadas, uso de protetor solar, utilização de chapéus e evitar o 
Sol ainda são as principais recomendações com relação à prevenção primária do câncer de pele em geral ${ }^{37}$. Os efeitos a curto prazo das medidas de proteção têm sido avaliados pela redução de freqüências de lesões precursoras para as doenças mais graves que podemos relacionar com a exposição solar 8,55,56.

Os resultados do nosso inquérito, envolvendo resultados de 16 cidades brasileiras, podem contribuir para o planejamento de estratégias diferenciadas de proteção à radiação solar no país, assim como estimular novos estudos que aprofundem o perfil de conhecimento sobre todos os elementos que fazem parte da cadeia complexa de exposição à radiação ultravioleta, propiciando o desenvolvimento de programas mais eficazes de prevenção de doenças e agravos relacionados à exposição solar.

\section{Resumo}

Este artigo explora a relação entre variáveis sócio-demográficas e comportamentais com proteção solar, levando-se em consideração as diferenças regionais existentes. Um inquérito de base populacional foi realizado em 15 capitais brasileiras e Distrito Federal, e as informações sobre exposição solar foram coletadas para um total de 16.999 indivíduos de 15 anos ou mais. Quando comparamos os individuos residentes nas regiões Norte e Sul do país, as diferenças brutas entre mulheres e homens nas proporções de utilização do protetor solar e do chapéu foram, respectivamente, para a Região Norte, +10,9\% (IC95\%: 7,1; 14,6) e-11,6\% (IC95\%: -17,0; -6,3), e Sul do país, +21,3\% (IC95\%: 17,7; 24,9) e-16,0\% (IC95\%: -20,2; -12,5). As diferenças ajustadas confirmaram que, tanto no Norte quanto no Sul do país, as mulheres mais freqüentemente utilizaram como proteção o filtro solar e menos freqüentemente referiram o uso de chapéu do que os homens. Contudo, essas diferenças não foram homogêneas entre as regiões (termos de interação $p<0,05$ ).

Luz Solar; Protetores de Raios Solares; Gênero

\section{Colaboradores}

A. S. Szklo efetuou os cálculos e as tabelas, discutiu os resultados e analisou-os junto à equipe. Redigiu o corpo do artigo, tendo trabalhado nele até a sua forma final. L. M. Almeida participou da discussão dos resultados, tendo colaborado na redação do artigo até a sua forma final. V. Figueiredo contribuiu na elaboração/preparação do artigo. J. A. Lozana colaborou na elaboração/ preparação da seção de material e métodos deste artigo. G. A. S Mendonça e L. Moura participaram da discussão dos resultados e da redação do artigo até a sua forma final. M. Szklo sugeriu plano de análise, participou das discussões dos resultados e das análises dos dados e colaborou na revisão inicial e final do texto dando sugestões.

\section{Agradecimentos}

O inquérito domiciliar sobre comportamentos de risco e morbidade referida de agravos e doenças não transmissíveis foi financiado pela Secretaria deVigilância em Saúde/MS com a contrapartida do Instituto Nacional de Câncer. As Secretarias de Estado de Saúde do Rio Grande do Norte, de Santa Catarina e de Sergipe e a Secretaria Municipal de Curitiba financiaram a coleta de dados em suas respectivas capitais. Gostaríamos também de agradecer ao Institute for Global Tobacco Control, Johns Hopkins Bloomberg School of Public Health e ao Fogarty International Center of the National Institutes of Health, nos Estados Unidos, pelo apoio à nossa pesquisa (grant R01-HL-73699). 


\section{Referências}

1. Gallagher RP, Lee TK. Adverse effects of ultraviolet radiation: a brief review. Prog Biophys Mol Biol 2006; 92:119-31.

2. Coordenação de Prevenção e Vigilância, Instituto Nacional de Câncer. Estimativa 2006: incidência de câncer no Brasil. Rio de Janeiro: Instituto Nacional de Câncer; 2005.

3. Oliveira WRP, Rady PL, Grady J, Hughes T, Neto CF, Rivitti EA, et al. Association of p53 arginine polymorphism with skin cancer. Int J Dermatol 2004; 43:489-93.

4. Bakos L, Wagner M, Bakos R, Leite CS, Sperhacke CL, Dzehaniak KS, et al. Sunburn, sunscreens, and phenotypes: some risk factors for cutaneous melanoma in southern Brazil. Int J Dermatol 2002; 41:557-62.

5. Maia M, Proença NG, Moraes JC. Risk factors for basal cell carcinoma: a case-control study. Rev Saúde Pública 1995; 29:27-37.

6. Wang LE, Xiong P, Strom SS, Goldberg LH, Lee JE, Ross MI, et al. In vitro sensitivity analysis to ultraviolet B light and skin cancer risk: a case-control analysis. J Natl Cancer Inst 2005; 97:1822-31

7. MacLennan R, Kelly JW, Rivers JK, Harrison SL. The eastern Australian childhood nevus study: site differences in density and size of melanocytic nevi in relation to latitude and phenotype. J Am Acad Dermatol 2003; 48:367-75.

8. Kwan TY, Belke TW, Enta T. Sex differences in the anatomical distribution of melanocytic nevi in Canadian Hutterite children. J Cutan Med Surg 2000; 4:58-62.

9. Azzarello LM, Dessureault S, Jacobsen PB. Sunprotective behavior among individuals with a family history of melanoma. Cancer Epidemiol Biomarkers Prev 2006; 15:142-5

10. Garbe C, Buettner PG. Predictors of the use of sunscreen in dermatological patients in Central Europe. Prev Med 2000; 31:134-39.

11. Elwood JM, Gallagher RP, Hill GB, Pearson JCG. Cutaneous melanoma in relation to intermittent and constant sun exposure-the western Canada melanoma study. Int J Cancer 1985; 35:427-33.

12. Maier T, Korting HC. Sunscreens - which and what for? Skin Pharmacol Physiol 2005; 18:253-62.

13. Lowe NJ. An overview of ultraviolet radiation, sunscreens, and photo-induced dermatoses. Dermatol Clin 2006; 24:9-17.

14. Bajdik CD, Gallagher RP, Hill GB, Fincham S. Sunlight exposure, hat use, and squamous cell skin cancer on the head and neck. J Cutan Med Surg 1998; 3:68-73.

15. Diffey BL, Cheeseman J. Sun protection with hats. Br J Dermatol 1992; 127:10-2.

16. Wong JC, Airey DK, Fleming RA. Annual reduction of solar UV exposure to the facial area of outdoor workers in Southeast Queensland by wearing a hat. Photodermatol Photoimmunol Photomed 1996; 12:131-5.
17. Coordenação de Prevenção e Vigilância, Instituto Nacional de Câncer. Inquérito domiciliar sobre comportamentos de risco e morbidade referida de doenças e agravos não transmissíveis: Brasil, 15 capitais e Distrito Federal, 2002-2003. Rio de Janeiro: Instituto Nacional de Câncer; 2004.

18. International Physical Activity Questionnaire. Scoring protocol. http://www.ipaq.ki.se/dloads/ Scoring\%20short\%20April04.pdf (acessado em 09/ Ago/2005).

19. Rao JNK, Scott AJ. On chi-squared tests for multiway contingency tables with cell proportions estimated from survey data. Annals of Statistics 1984; 12:46-60.

20. Szklo M, Nieto FJ. Epidemiology beyind the basics. Gaithersburg: Aspen Publishers; 2000.

21. Skinner CJ, Holt D, Smith TMF. Analysis of complex surveys. New York: Wiley and Sons; 1989.

22. Ramirez CC, Federman DG, Kirsner RS. Skin cancer as an occupational disease: the effect of ultraviolet and other forms of radiation. Int J Dermatol 2005; 44:95-100.

23. Guénel P, Laforest L, Cyr D, Févotte J, Sabroe S, Dufour $\mathrm{C}$, et al. Occupational risk factors, ultraviolet radiation, and ocular melanoma: a case-control study in France. Cancer Causes Control 2001; 12:451-9.

24. Cardinez CJ, Cokkinides VE, Weinstock MA, O'Connell MC. Sun protective behaviors and sunburn experiences in parents of youth ages 11 to 18 . Prev Med 2005; 41:108-17.

25. Reynolds KD, Blaum JM, Penélope J, Jester BSN, Weiss H, Soong SJ, et al. Predictors of sun exposure in adolescents in a southeastern U.S. population. J Adolesc Health 1996; 19:409-15.

26. Alberg AJ, Herbst RM, Genkinger JM, Duszynski KR. Knowledge, attitudes, and behaviours toward skin cancer in Maryland youths. J Adolesc Health 2002; 31:372-7.

27. Bataille V, Winnett A, Sasieni P, Newton BJA, Cuzick J. Exposure to the sun and sunbeds and the risk of cutaneous melanoma in the UK: a case-control study. Eur J Cancer 2004; 40:429-35.

28. Aquilina S, Gauci AA, Ellul M, Scerri L. Sun awareness in Maltese secondary school students. J Eur Acad Dermatol Venereol 2004; 18:670-5.

29. Benvenuto-Andrade C, Zen B, Fonseca G, De Villa D, Cestari T. Sun exposure and sun protection habits among high-school adolescents in Porto Alegre, Brazil. Photochem Photobiol 2005; 81:630-5.

30. Harrison RA, Haque AU, Roseman JM, Soong SJ. Socioeconomic characteristics and melanoma incidence. Ann Epidemiol 1998; 8:327-33.

31. Woodward A, Boffetta P. Environmental exposure, social class, and cancer risk. IARC Sci Publ 1997; (138):361-7.

32. de Vries H, Lezwijn J, Hol M, Honing C. Skin cancer prevention: behaviour and motives of Dutch adolescents. Eur J Cancer Prev 2005; 14:39-50.

33. Hall HI, May DS, Lew RA, Koh HK, Nadel M. Sun protection behaviors of the U.S. white population. Prev Med 1997; 26:401-7. 
34. McCarthy EM, Ethridge KP, Wagner RF. Beach holiday sunburn: the sunscreen paradox and gender differences. Cutis 1999; 64:37-42.

35. Kricker A, Armstrong BK, English DR, Heenan PJ. Does intermittent sun exposure cause basal cell carcinoma? A case control study in western Australia. Int J Cancer 1995; 60:489-94.

36. Oncology Cooperative Group of the Italian Group for Epidemiologic Research in Dermatology (GISED). Improving sun protection behaviour in children: study design and baseline results of a randomized trial in Italian elementary schools. The 'Sole Si Sole No' GISED project. Dermatology 2003; 207:291-7.

37. Stanton WR, Janda M, Baade PD, Anderson P. Primary prevention of skin cancer: a review of sun protection in Australia and internationally. Health Promot 2004; 19:369-78.

38. Crane LA, Schneider LS, Yohn JJ, Morelli JG, Plomer KD. "Block the sun, not the fun": evaluation of a skin cancer prevention program for child care centers. Am J Prev Med 1999; 17:31-7.

39. Sullivan SS, Rosen CJ, Halteman WA, Chen TC, Holick MF. Adolescent girls in Maine at risk for vitamin D insufficiency. J Am Diet Assoc 2005; 105:971-4.

40. Feldman SR, Liguori A, Kucenic M, Rapp SR, Fleischer $\mathrm{AB}$, Lang $\mathrm{W}$, et al. Ultraviolet exposure is a reinforcing stimulus in frequent indoor tanners. J Am Acad Dermatol 2004; 51:45-51.

41. Koblenzer CS. The psychology of sun-exposure and tanning. Clin Dermatol 1998; 16:421-8.

42. Glanz K, Shigaki D, Maddock JE, Isnec MR. A randomized trial of skin cancer prevention in aquatics settings: the pool cool program. Health Psychol 2002; $21: 579-87$

43. Horsley L, Charlton A, Waterman C. Current action for skin cancer risk reduction in English schools: pupils' behavior in relation to sunburn. Health Educ Res 2002; 17:715-31.

44. Hall HI, Jones SE, Saraiya M. Prevalence and correlates of sunscreen use among US high school students. J Sch Health 2001; 71:453-7.

45. Elwood JM, Difey BL. A consideration of ambient solar ultraviolet radiation in the interpretation of studies of the aetiology of melanoma. Melanoma Res 1993; 3:113-22.
46. Fitzpatrick TB. The validity and practicality of sunreactive skin types I through VI. Arch Dermatol 1988; 124:869-71.

47. Diffey BL, Elwood JM. Tables of ambient solar ultraviolet radiation for use in epidemiological studies of malignant melanomas and other diseases. In: Gallagher RP, Elwood JM, editors. Epidemiological aspects of cutaneous malignant melanoma. Boston: Kluwer; 1994. p. 81-105.

48. Branstrom R, Kristjansson S, Ullén H. Risk perception, optimistic bias, and readiness to change sun related behaviour. Eur J Public Health 2005; 29: 1-6.

49. Berret J, Liardet S, Scaletta C, Panizzon R, Hohlfeld P, Applegate LA. Use of sunscreens in families living in Switzerland. Dermatology 2002; 204:202-8.

50. Ferrini RL, Perlman M, Hill L. American College of Preventive Medicine practice policy statement: skin protection from ultraviolet light exposure. Am J Prev Med 1998; 14:83-6.

51. Instituto Brasileiro de Geografia e Estatística. Anuário estatístico - censo demográfico de 1991. Brasília: Instituto Brasileiro de Geografia e Estatística; 1997.

52. Green A, Martin NG. Measurement and perception of skin colour in a skin cancer survey. Br J Dermatol 1990; 123:77-84.

53. Sies H, Stahl W. Nutritional protection against skin damage from sunlight. Annu Rev Nutr 2004; 24:173-200.

54. Broekmans WM, Vink AA, Boelsma E, KloppingKetelaars WA, Tijburg LB, Veer P, et al. Determinants of skin sensitivity to solar radiation. Eur J Clin Nutr 2003; 57:1222-9.

55. Lee TK, Rivers JK, Gallagher MA. Site-specific protective effect of broad-spectrum sunscreen on nevus development among white schoolchildren in a randomized trial. J Am Acad Dermatol 2005; 52:786-92.

56. Rocha FP, Menezes AMB, Almeida Junior HL, Tomasi E. Marcadores e fatores de risco para queratoses actínicas e carcinomas basocelulares: um estudo de caso-controle. An Bras Dermatol 2004; 79:441-54.

Recebido em 05/Out/2005

Versão final reapresentada em 25/Abr/2006

Aprovado em 30/Out/2006 